significantly. Though the Fearnley classification showed a floor effect, our method related to function more linearly (R-squared: 0.42). Each parameter of our method showed statistical significance for the clusters by regression analysis. And the ratio of the partial regression coefficient of each parameter was around 1:1:3:2, so we calculated a 'cluster score' using a weighted score for each parameter. A regression analysis has shown a strong correlation $(r=0.95, p<0.001)$; however, its scatter plot also suggested that it is difficult to classify the cluster using CS only. We found one type of hand in which bone destruction precedes the joint dislocation and one type in which joint dislocation progresses with little deviation during UD progression. It is considered that two patterns of pathological findings and included some suggested indications for operative therapy to recover disability due to UD.

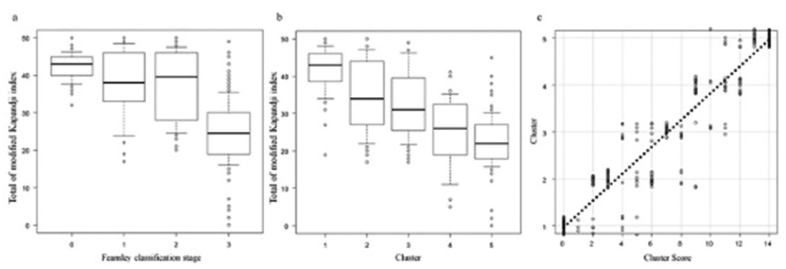

Conclusions: Our UD evaluation method is quite simple but is closely related to function. Additionally, it enables dividing UD hands into five stages. Thus, our assessment should be beneficial compared to the Fearnley classification in considering treatments of UD.

Acknowledgements: The authors thank all the researchers involoved in this 10 year observational study.

Disclosure of Interest: None declared

DOI: 10.1136/annrheumdis-2018-eular.1496

\section{THU0705 OCCURRENCE OF ANKYLOSING SPONDYLITIS (AS) AMONG RELATIVES OF PROBANDS WITH RADIOGRAPHIC AS AND NON-RADIOGRAPHIC AS/ AXIAL SPONDYLOARTHRITIS (SPA)}

S. Van Der Linden ${ }^{1}$, P. Villiger ${ }^{2}$, H. Baumberger ${ }^{3}$, M. Brown ${ }^{4}$, A. Boonen ${ }^{1}, M$ A. Khan ${ }^{5} .{ }^{1}$ Rheumatology, University Medical Center, Maastricht, Netherlands; ${ }^{2}$ Rheumatology, Inselspital, University of Bern, Bern; ${ }^{3}$ NA, Flims, Switzerland; ${ }^{4}$ Health and Biomedical Innovation, Queensland University of Technology, Brisbane, Australia; ${ }^{5}$ Rheumatology, Case Western Reserve University, Cleveland, $\mathrm{OH}$, USA

Background: The occurrence of AS in families as it relates to the presence or absence of HLA-B27 and radiographic sacroiliitis is not well-known.

Objectives: To assess among relatives the occurrence of $A S$ and its association with HLA-B27 and presence of sacroiliitis among AS probands. AS is broader than just radiographic (classical) AS by modified New York criteria (mNY). We asked whether the likelihood of having a relative with classical AS is comparable for probands with radiographic $\mathrm{AS}$ by $\mathrm{mNY}$ criteria, and probands with non-radiographic AS/axSpA.

Methods: Re-analysis of the 1985-86 Swiss AS Family Study: 1178 subjects (363 clinically defined AS probands, 806 first-degree relatives, and 9 spouses) had participated. The AS patients were members of the Swiss AS patient society. The invited and consenting participants underwent physical exam of the spine and joints, completed questionnaires dealing with musculoskeletal problems, had pelvic radiographs taken, and provided blood samples for HLA-A,B,C typing. Radiographs were blindly read twice by each of up to 4 experienced readers. They scored sacroiliac joints according to NY criteria for sacroiliitis (bilateral grade $\geq 2$ or unilateral grade $\geq 3$ ). The study had been approved by the ethical committee of the University of Bern, Switzerland.

Results: Among 358 AS probands, 308 (86\%) were HLA-B27(+) and 50 (14\%) lacked this gene. The radiographic NY criteria were met by $81 \%$ of the $308 \mathrm{HLA}$ B27(+) probands versus only $44 \%$ of the $50 \mathrm{HLA}-\mathrm{B} 27(-)$ probands. AS, as defined by NY criteria, was observed only among relatives of HLA-B27(+) probands; and all 14 of these relatives were also HLA-B27(+). Note: The probands in 12 of these 14 cases met the NY criteria, while 2 did not. Two of 59 HLA-B27(+) relatives from AS probands not-fulfilling the $\mathrm{mNY}$ criteria met the NY criteria, compared with 12 of $245 \mathrm{HLA}-\mathrm{B} 27(+)$ relatives from AS probands fulfilling the $\mathrm{mNY}$ criteria $(p=0.62)$. Interestingly, inflammatory back pain (by Calin criteria) occurred among 46 of 286 (16.1\%) HLA-B27(+) relatives of HLA-B27(+) probands, but in only 27 of 272 (9.9\%) HLA-B27(-) relatives of $\mathrm{HLA}-\mathrm{B} 27(+)$ probands $(\mathrm{p}=0.031)$

Conclusions: AS among relatives was only observed in families of HLA-B27(+) AS/axSpA probands. The risk for HLA-B27(+) relatives to develop AS by NY criteria seems not to be influenced by the presence of sacroiliitis in the HLA-B27(+) AS/axSpA probands. HLA-B27(+) relatives who do not show radiographic sacroiliitis are at higher risk to demonstrate symptoms of non-radiological AS/ axSpA as they seem more prone to have inflammatory back pain

\section{REFERENCE:}

[1] Khan MA, van der Linden SM, Kushner I, Valkenburg HA, Cats A. Spondy litic disease without radiologic evidence of sacroiliitis in relatives of HLA B27 positive ankylosing spondylitis patients. Arthritis Rheum 1985;28 (1):40-3.

Disclosure of Interest: None declared DOI: 10.1136/annrheumdis-2018-eular.5583

\section{THU0706 \\ PRESENCE OF EXTRACTABLE NUCLEAR ANTIGENS (ENA) ANTIBODIES IN A LARGE POPULATION-BASED COHORT FROM THE NETHERLANDS}

S. Arends ${ }^{1}$, L. Brouwer ${ }^{1}$, C. Roozendaal ${ }^{2}$, B. Doestzada ${ }^{1}$, H. Bootsma ${ }^{1}$, K. de Leeuw ${ }^{1} .{ }^{1}$ Rheumatology and Clinical Immunology; ${ }^{2}$ Laboratory Medicine, UMCG, Groningen, Netherlands

Background: Previous studies have demonstrated that years before the clinical onset of auto-immune diseases, auto-antibodies can already be present in the blood of these patients. However, it is also known that some individuals can have these auto-antibodies and will never develop an auto-immune disease. So far, little information is available on the presence of Extractable Nuclear Antigens antibodies (anti-ENA) in the general population in relation to the risk of developing an auto-immune disease.

Objectives: To estimate the prevalence of anti-ENA and their association with the presence of known risk factors of Systemic Lupus Erythematosus (SLE) and Sjögren Syndrome (SS) in the general Dutch population.

Methods: Lifelines is a prospective population-based cohort study in the Netherlands. Cross-sectional data from 40135 participants were analysed. The detec tion of anti-ENA was performed using the ENA-CTD (connective tissue disease) screen on the Phadia-250 analyser with a ratio $>1.0$ are considered positive. An extensive questionnaire was taken on demographic and clinical information (e.g. early musculoskeletal symptoms). Furthermore, some general blood parameters were available. SLE and SS were defined by a combination of self-reported SLE or SS, specific medication use and visiting a medical specialist within the last year. Characteristics were compared between 3 groups: SLE/SS patients, antiENA positive, and anti-ENA negative participants (without defined SLE/SS).

Results: Of the total 40135 consecutive individuals, 41 were detected as having defined SLE or SS of whom $48 \%$ were anti-ENA positive SLE/SS patients were older and more often female. Of the remaining individuals, ${ }^{40,094} 1089$ (2.7\%) were anti-ENA positive and anti-ENA positivity was also significantly associated with older age and female gender in this population. No significant associations were found for smoking and alcohol intake. As might be expected, SLE/SS patients reported more often complaints concerning fatigue, joint pain and joint stiffness. But also anti-ENA-positive participants reported significantly more often joint stiffness compared to anti-ENA-negative participants. Interestingly, levels of haemoglobin, leucocytes and lymphocytes were significantly decreased in anti-ENApositive participants compared to anti-ENA-negative participants.

Conclusions: In this large population-based study, the prevalence of anti-ENA positivity was $2.8 \%$ for the total group and $2.7 \%$ when excluding patients with SLE or SS. Older age, female gender, joint complaints and lower levels of haemoglobin, leucocytes and lymphocytes were more frequently present in anti-ENA-positive participants. Longitudinal studies are performed up to 15 years to investigate which individuals might develop SLE or SS to be able to develop prediction models.

Disclosure of Interest: None declared

DOI: 10.1136/annrheumdis-2018-eular.4606

\section{THU0707 ANALYSIS OF ANTINUCLEAR ANTIBODIES IN BREAST CANCER PATIENTS}

A. Neppel, M.C.C. Machoski, T. A. F. G. Santos, R. Nisihara, T.L. Skare Departamento de Reumatologia, Hospital Universitário Evangélico de Curitiba, Curitiba, Brazil

Background: Analysis of antinuclear antibodies (ANA) may be found in malignant diseases

Objectives: To study the prevalence of ANA in breast cancer patients and its association with tumour characteristics.

Methods: Ninety one patients with breast mass detected by image studies and assigned to do diagnostic biopsy and eventual surgical treatment were studied for demographical, tumour data and presence of ANA. Serum of positive ANA patients was submitted to examination of ENA (extractable nuclear antigens) 